\title{
Combined Corneal Wedge Resection And Corneal Cross-Linking For Pellucid Marginal Degeneration: A First Report
}

This article was published in the following Dove Press journal:

Therapeutics and Clinical Risk Management

\section{George Kymionis \\ Nafsika Voulgari \\ Erwin Samutelela \\ George Kontadakis (iD \\ David Tabibian}

Ophthalmology Department, University of Lausanne, Jules Gonin Eye Hospital, Lausanne, Switzerland
Correspondence: George Kymionis Hôpital Ophtalmique Jules Gonin, 15 avenue de France, Lausanne 1004 Switzerland

Tel +4I793332259

Email gkymionis@gmail.com
Background: Advanced pellucid marginal degeneration is a debilitating disease that warrants the use of surgery when the visual acuity is reduced and contact lenses are not tolerated anymore. It is traditionally managed with corneal transplantation, however alternative surgical options exist. Corneal wedge resection allows for good visual rehabilitation without the risks of tissue rejection. However topographical and refractive results are in some instance fluctuating. We present here the use of corneal cross-linking in order to stabilize the parameters on the long term.

Case presentation: We present here the case of a 53 years old patient with bilateral advanced pellucid marginal degeneration. As he is now intolerant to contact lenses a surgical option is offered to him. In order to avoid using donated tissue through corneal grafting we decide to perform a sectorial lamellar crescentric wedge excision of the thinner inferior part of the cornea involving the pellucid marginal degeneration and suture it. The first eye shows initial good results however after few months regression is observed. The second eye is then treated with the same surgical technique combined with cornea cross-linking. Long-term follow-up shows stabilization and absence of regression in the second eye up to eight months after the surgery.

Conclusion: Combining corneal cross-linking with corneal wedge resection in the case of advanced pellucid marginal degeneration patients could be a good option in order to stabilize topographical and refractive results and reduces the risk of regression.

Keywords: cornea, ectasia, pellucid marginal degeneration, cornea cross-linking, wedge resection

\section{Introduction}

Pellucid marginal degeneration (PMD) is a rare non-inflammatory corneal ectatic disorder that is characterized by progressive peripheral corneal thinning. The disease progression can induce irregular astigmatism leading sometimes to reduced visual acuity. PMD is distinctive in its features from keratoconus in the way that there is a crescent-shaped inferior band of corneal thinning with associated ectasia and the disease is usually diagnosed in an older set of patients with a male predilection. ${ }^{1}$ Some cases of PMD can overlap keratoconus and typical patterns such as "crab-claws" or "kissing birds" patterns can be seen in both diseases as initially described. Modern topographical measurement devices such as Scheimpflug imaging allow us to distinguish now crab-claw patterns in PMD-like keratoconus from rarer true PMD disease. Reduced visual acuity can be initially compensated by contact lenses such as rigid gas permeable or scleral contact lenses. 
However when those fail to provide adequate correction due to significant astigmatism or are not tolerated anymore, surgery can be considered. Standard surgical management usually includes penetrating keratoplasty or deep anterior lamellar keratoplasty. Nonetheless the peripheral inferior thinning and steepening of the cornea moderates the success of such surgery. Marked irregular astigmatism can be induced by suturing the graft to a thin peripheral tissue and larger grafts that could overcome this issue present a larger risk of graft rejection. ${ }^{2}$ Thus the alternative surgical option of crescentric corneal wedge resection has been proposed some years ago with good results on visual rehabilitation and sparring the use of donated corneal tissue. However in some cases topographical regression is observed with consecutive reduced visual acuity. ${ }^{3,4}$

\section{Methods}

We present here the case of a patient treated on the first eye with the already described procedure that later on presented topographical regression and treated for the second eye with a combination of surgery and corneal crosslinking to stabilize refractive and topographical results.

\section{Case Presentation}

A 53 years old gentleman was referred to our Ophthalmology department for progressive bilateral pellucid marginal degeneration. He was known for a previous benign traumatic corneal abrasion in his right eye 14 years ago with no other relevant ocular history. He was previously fitted with scleral contact lenses with good visual improvement however he later stopped using them as he experienced intolerance despite multiple trials. He has been followed with corneal topography that demonstrated progressive increase in astigmatism over the past year.

At his first clinical examination uncorrected for distance visual acuity (UDVA) was 0.05 (decimal) in the right eye and counting fingers in the left eye. Corrected for distance visual acuity (CDVA) was 0.08 in the right eye with $-3.00-5.25 / 76^{\circ}$ and 0.08 in the left eye with -11.75 $-7.50 / 160^{\circ}$. Slit lamp examination showed mild posterior blepharitis, bilateral clear corneas with visible inferior protrusion and associated thinning with an otherwise quiet and deep anterior chamber. Intraocular pressure was $9 \mathrm{mmHg}$ in both eyes with non-contact measurements, crystalline lens had no signs of cataract bilaterally, and fundus examination was within normal limits.

Keratometric readings were obtained from Scheimpflug imaging (Pentacam, Oculus, Optikgeräte GmbH, Wetzlar,
Germany) in both eyes. Both topographies showed PMD patterns with the steepest anterior point inferiorly displaced with associated thinning and irregular astigmatism on anterior sagittal maps (Figures 1A and 2A). Anterior segment optical coherence tomography (AS-OCT) (Optovue, Inc., Freemont, CA) confirmed the inferior thinning in both eyes with a thinnest measurement of $341 \mu \mathrm{m}$ in the right eye and $270 \mu \mathrm{m}$ in the left eye.

As the patient reported reduced vision bilaterally with markedly reduced quality of life and presented persisting contact lens intolerance despite numerous trials, a surgical option was offered in order to improve the patient's visual acuity. Instead of a penetrating keratoplasty or a deep anterior lamellar keratoplasty we decided to perform a sectorial lamellar crescentric wedge resection of the thinner inferior part of the cornea and suture it. Risks and benefits were explained to the patient, consent obtained and the surgery was scheduled under general anesthesia.

The left eye was prepped and draped in a sterile manner. The epithelium was removed with a hockey stick inferiorly over the thinner crescentric part of the cornea. One side port was created at 2 o'clock and a cohesive viscoelastic was injected in the anterior chamber in order to maintain a stable chamber in case of perforation. A circular blade was used to create a crescentric incision on the lower part of the cornea starting from 9 o'clock up to 3 o'clock. The cornea was then dissected as close as possible to the Descemet membrane and the crescentric dissection was then excised. The superior corneal margin of the excised tissue was then pulled down and sutured with seven interrupted 10-0 nylon sutures to the lower margin. The viscoelastic was removed and an antibiotic ointment was added with an eyepad.

On the following day examination, his UDVA in the left eye had improved to 0.5 and the CDVA to 0.6. Slit-lamp examination showed a clear central cornea. The peripheral inferior cornea showed tight sutures and no residual thinning. The rest of the slit-lamp examination was within normal limits and intraocular pressure was $10 \mathrm{mmHg}$ in the right eye and $7 \mathrm{mmHg}$ in the left eye. The corneal astigmatism was also regularized with regular oblique astigmatism pattern on the anterior sagittal map with $10.9 \mathrm{D}$ at $52^{\circ}$ axis. The thinnest pachymetry on Scheimpflug imaging had improved to $470 \mu \mathrm{m}$ (Figure 1B).

The patient was followed monthly with topography and early signs of regression were evident in his second month follow up in comparison to first month. Four months afterwards and following corneal sutures removal, the patient 


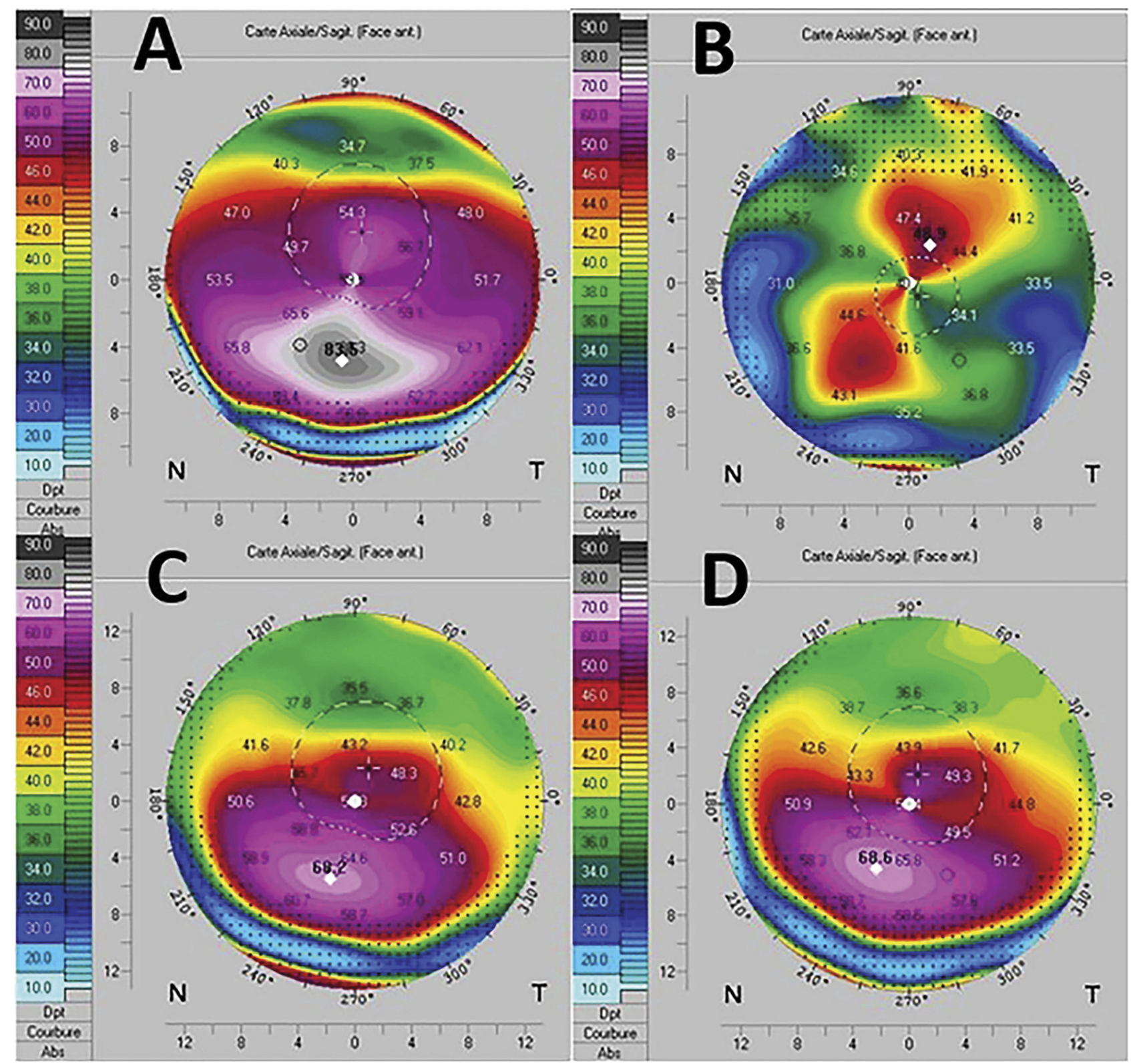

Figure I Scheimpflug-based corneal topography of the left eye (OS). (A) Preoperatively, demonstrating PMD pattern with inferior steepening associated with thinning and irregular astigmatism on the anterior sagittal map. (B) At day one following crescentic corneal wedge resection, showing regularization of the corneal astigmatism. (C) Four months postoperatively, showing marked steepening inferiorly. (D) Ten months postoperatively, showing recurring irregular astigmatism in the anterior sagittal map coinciding with inferior steepening and a minimum corneal thickness of $387 \mu \mathrm{m}$.

reported an improvement in his vision: UDVA is 0.5 and improves to 1.0 with refraction. The anterior sagittal topographical pattern on topography is however more irregular at this follow-up showing regression of the treatment with marked steepening inferiorly and loss of regular pattern (Figure 1C). Ten months after the surgery the patient's left eye UDVA has then reduced to 0.05 improving to 0.2 CDVA. On scheimpflug imaging the anterior sagittal map showed recurring irregular astigmatism with inferior marked steepening (Figure 1D).
The second eye was planned for surgery two months after the first one. As early signs of regression were observed at the two months examination on the first eye topography it was decided to perform again a corneal wedge resection and combine it with corneal cross-linking in order to stabilize postoperative results and reduce the risk of regression. Surgery was performed in the right eye similarly as described above for the left eye. At the end of the surgery an epithelium-off corneal cross-linking was performed with $9 \mathrm{mw} / \mathrm{cm} 2$ for $10 \mathrm{mins}(5.4 \mathrm{~mJ} / \mathrm{cm} 2$ 

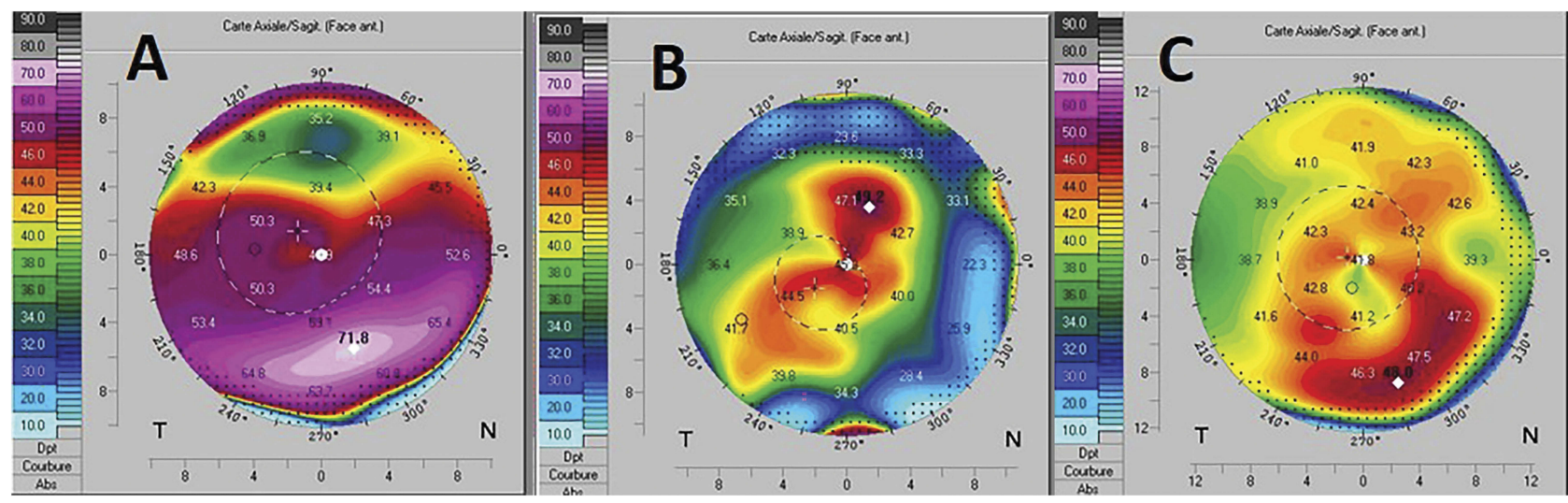

Figure 2 Scheimpflug-based corneal topography of the right eye (OD). (A) Preoperatively, demonstrating PMD pattern with inferior steepening associated with thinning and irregular astigmatism on the anterior sagittal map. (B) Four days following combined corneal wedge resection and corneal cross-linking, showing regularization of the topographic pattern. (C) Eight months postoperatively, depicting a stable regularized anterior pattern with mild inferior steepening and a thinnest pachymetry of $452 \mu \mathrm{m}$.

fluence) settings. Corneal thickness at the area of the suturing was measured with ultrasound pachymetry prior to irradiation and thinnest measurement was $435 \mu \mathrm{m}$. The epithelium was removed on the inferior half of the cornea involving the disease beforehand. The irradiation circle included the area of the suturing. Postoperative care was similar as for the first eye.

Four days after the surgery the UDVA was 0.5 . Treated cornea showed tight sutures, a healing epithelial defect without any sign of infection and within normal limits intraocular pressure. Topographical patterns were regularized with now oblique $3.7 \mathrm{D}$ anterior astigmatism at $25^{\circ}$ (Figure 2B). One month postoperatively demarcation line depth was measured at $250 \mu \mathrm{m}$. Sutures were removed up to 4 months postoperatively. Eight months after the surgery UDVA is 0.25 and improves to 0.6 CDVA on refraction. Scheimpflug imaging shows a stable regularized anterior pattern within the $4.5 \mathrm{~mm}$ central cornea with $2.3 \mathrm{D}$ astigmatism, inferiorly a mild steepening is present (Figures 2C, 3A and B). At the 14th postoperative month visit, his CDVA remained 0.6 and corneal topography demonstrated a stable pattern in comparison to previous follow up visits.

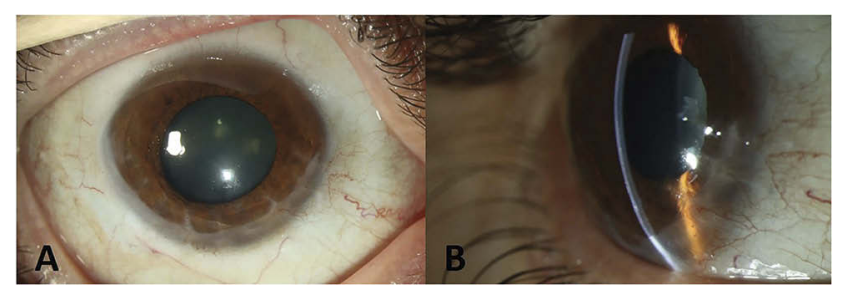

Figure 3 Post-operative slit-lamp photography of anterior segment of the right eye with broad illumination $(\mathbf{A})$ and with slit illumination from side view (B).

\section{Discussion}

Several surgical options exist to treat pellucid marginal degeneration. ${ }^{5}$ Penetrating keratoplasty as well as deep anterior lamellar keratoplasty are widely used ones in advanced cases and aim to restor visual acuity through reduction of corneal astigmatism with donated tissue. Corneal cross-linking has been used for the stabilization of the disorder either alone or combined with laser ablation for the imrovement of visual acuity. ${ }^{6,7}$ Studies demonstrate efficacy of the technique for halting progression although there is a lack of large series with long term follow up. Lately, sliding keratoplasty in combination with cross-linking has also been presented. ${ }^{8}$ Corneal wedge resection was initially described by Durand et al in 1991 as an alternative to treat pellucid marginal degeneration. ${ }^{4}$ However, this sectoral crescentric removal of the diseased tissue has only been described in a limited number of reports in the litterature. ${ }^{9-11}$ Sectoral removal of the abnormal ectatic tissue is a useful surgical approach that permits to reduce or even avoid complications linked to grafted tissue such as rejection, primary or secondary failure and glaucoma. Furthermore, the superiorly located "healthy" corneal tissue is pulled down towards the central visual axis and reduces the overall corneal astigmatism through this "face-lift" of the cornea and excision of the thinned pathological tissue. ${ }^{12}$ Cornea remodeling and wound healing takes place after the surgery and induces progressive changes in corneal topography. Selective suture removal can help shaping this corneal remodelling in order to stabilize or improve topographical changes before achieving refractive stability. In some instance however topographical instability and regression of the treatment will take place despite good initial surgical outcome and following management. ${ }^{2,3}$ 
In our case the visual acuity improved quickly after the surgery of the first eye at day one associated with regularization of the corneal astigmatism on topography. However two months after the surgery early irregular changes were observed on the topography. Despite an overrall improved topography compared to preoperative measurements, the visual acuity was reduced at the ten months follow-up with partial regression of the treatment visible on Scheimpflug imaging. Rigid contact lenses can help to partially compensate the recurring irregular astigmatism. In our case however this was not an option as the patient was intolerant to contact lenses. Corneal crosslinking was thus successfully used to help stabilize corneal healing in the second eye with good refractive and topographical outcomes.

Corneal cross-linking is nowadays used as the standard of care to stabilize progressive ectatic disorders such as keratoconus with good long-term outcomes. ${ }^{13,14}$ It has since then been used for several other purposes such as infectious keratitis or more recently in donor tissue for penetrating keratoplasties. In the later, results in animal and human model showed both that surgically induced astigmatism is reduced postoperatively. ${ }^{15,16}$ In the randomized controlled trial by Huang and colleagues patient's with cross-linked donated corneas showed significantly improved visual acuity, lesser degree of spherical equivalent, lower $\mathrm{K}_{\max }$ and keratometric astigmatism compared to the non cross-linked group at three years follow-up. ${ }^{16}$ Interestingly Mukherjee et al reported also significant improvement in their animal experiment. ${ }^{15}$ Furthermore in a letter to the editor their group also proposed that the significant reduced higher-order aberrations observed in the animal cross-linked group could explain the improved visual acuity reported in the human study; however these parameters were not measured in the later study.

A possible limitation of the presented method is the questionable efficacy of corneal crosslinking in advanced cases of ectasia, such as those cases of pellucid marginal degeneration that are suitable for wedge resection. Other options other than grafting or wedge resection are suitable for earlier cases that can be combined with CXL. Nevertheless, this report showcases good results that a large series with long term follow up would be necessary to prove.

\section{Conclusion}

In contact-lens intolerant patients this surgery is a good alternative to more invasive ones such as penetrating keratoplasty or deep anterior lamellar surgery. A quick visual recovery can be expected as the healthy tissue is pulled within the central visual axis and allows good visual rehabilitation in patients with otherwise poor visual acuity. According to our results we propose here that the stiffening effect of corneal cross-linking helps also to stabilize topographical and refractive results and reduces the risk of regression in a deep anterior crescentric wedge excision for advanced pellucid marginal degeneration as it can do it for penetrating keratoplasties.

\section{Abbreviations}

CDVA, corrected for distance visual acuity; AS-OCT, anterior segment optical coherence tomography; UDVA, uncorrected for distance visual acuity; PMD, Pellucid marginal degeneration.

\section{Ethics Approval And Consent}

The involved patient gave his written consent to this scientific publication. No formal ethical committee approval was needed (Commission cantonale d'éthique de la recherché sur l'être humain, canton de Vaud). This complies with national and local guidelines, available reference on: http://www.cervd.ch/soumission/premiers-pas.html. Patient's consent has been obtained for publication of this manuscript.

\section{Data Sharing Statement}

The datasets of this report are available from the corresponding author on request.

\section{Author Contributions}

All authors contributed to data analysis, drafting or revising the article, gave final approval of the version to be published, and agree to be accountable for all aspects of the work.

\section{Disclosure}

The authors report no conflicts of interests in this work.

\section{References}

1. Shimazaki J, Maeda N, Hieda O, et al. National survey of pellucid marginal corneal degeneration in Japan. Jpn $J$ Ophthalmol. 2016;60:341-348. doi:10.1007/s10384-016-0462-0

2. Genc S, Cakir H, Guler E, Calli U. Refractive and corneal aberrometric changes after crescentic lamellar wedge resection in pellucid marginal degeneration. Eye Contact Lens. 2017;44:S76-S80.

3. MacLean H, Robinson LP, Wechsler AW. Long-term results of corneal wedge excision for pellucid marginal degeneration. Eye (Lond). 1997;11(Pt 5):613-617. 
4. Duran JA, Rodriguez-Ares MT, Torres D. Crescentic resection for the treatment of pellucid corneal marginal degeneration. Ophthalmic Surg. 1991;22:153-156.

5. Moshirfar M, Edmonds JN, Behunin NL, Christiansen SM. Current options in the management of pellucid marginal degeneration. J Refract Surg. 2014;30:474-485. doi:10.3928/108159 7X-20140429-02

6. Hassan Z, Nemeth G, Modis L, Szalai E, Berta A. Collagen crosslinking in the treatment of pellucid marginal degeneration. Indian $J$ Ophthalmol. 2014;62:367-370. doi:10.4103/0301-4738.109523

7. Kymionis GD, Karavitaki AE, Kounis GA, Portaliou DM, Yoo SH, Pallikaris IG. Management of pellucid marginal corneal degeneration with simultaneous customized photorefractive keratectomy and collagen crosslinking. J Cataract Refract Surg. 2009;35:1298-1301. doi:10.1016/j.jcrs.2009.03.025

8. Spadea L, Maraone G, Cagini C. Sliding keratoplasty followed by transepithelial iontophoresis collagen cross-linking for pellucid marginal degeneration. J Refract Surg. 2016;32:47-50. doi:10.3928/ 1081597X-20151119-02

9. Busin M, Kerdraon Y, Scorcia V, Zambianchi L, Matteoni S. Combined wedge resection and beveled penetrating relaxing incisions for the treatment of pellucid marginal corneal degeneration. Cornea. 2008;27:595-600. doi:10.1097/ICO.0b013e318166c40c
10. Javadi MA, Karimian F, Hosseinzadeh A, Noroozizadeh HM, Sa'eedifar MR, Rabie HM. Lamellar crescentic resection for pellucid marginal corneal degeneration. J Refract Surg. 2004;20:162-165.

11. Cameron JA. Results of lamellar crescentic resection for pellucid marginal corneal degeneration. Am J Ophthalmol. 1992;113:296302. doi:10.1016/s0002-9394(14)71582-x

12. Maccheron LJ, Daya SM. Wedge resection and lamellar dissection for pellucid marginal degeneration. Cornea. 2012;31:708-715. doi:10.1097/ICO.0b013e31824000e3

13. Wollensak G, Spoerl E, Seiler T. Riboflavin/ultraviolet-a-induced collagen crosslinking for the treatment of keratoconus. Am J Ophthalmol. 2003;135:620-627. doi:10.1016/s0002-9394(02)02220-1

14. Raiskup F, Theuring A, Pillunat LE, Spoerl E. Corneal collagen crosslinking with riboflavin and ultraviolet-A light in progressive keratoconus: ten-year results. J Cataract Refract Surg. 2015;41:4146. doi:10.1016/j.jcrs.2014.09.033

15. Mukherjee A, Hayes S, Aslanides I, Lanchares E, Meek KM. Donor crosslinking for keratoplasty: a laboratory evaluation. Graefes Arch Clin Exp Ophthalmol. 2015;253:2223-2228. doi:10.1007/s00417-015-3160-6

16. Huang T, Ye R, Ouyang C, Hou C, Hu Y, Wu Q. Use of donors predisposed by corneal collagen cross-linking in penetrating keratoplasty for treating patients with keratoconus. Am J Ophthalmol. 2017;184:115-120. doi:10.1016/j.ajo.2017.10.003
Therapeutics and Clinical Risk Management

\section{Publish your work in this journal}

Therapeutics and Clinical Risk Management is an international, peerreviewed journal of clinical therapeutics and risk management, focusing on concise rapid reporting of clinical studies in all therapeutic areas, outcomes, safety, and programs for the effective, safe, and sustained use of medicines. This journal is indexed on PubMed Central, CAS,

\section{Dovepress}

EMBase, Scopus and the Elsevier Bibliographic databases. The manuscript management system is completely online and includes a very quick and fair peer-review system, which is all easy to use. Visit http://www.dovepress.com/testimonials.php to read real quotes from published authors. 